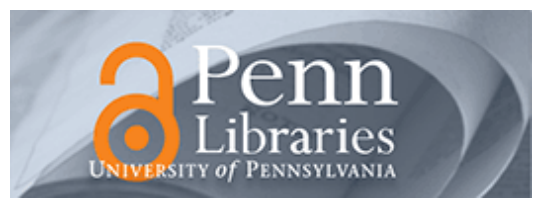

University of Pennsylvania ScholarlyCommons

September 2006

\title{
Large negative magnetoresistance and strong localization in highly disordered electrospun pregraphitic carbon nanofiber
}

\author{
Yu Wang \\ University of Pennsylvania \\ Jorge J. Santiago-Aviles \\ University of Pennsylvania, santiago@seas.upenn.edu
}

Follow this and additional works at: https://repository.upenn.edu/ese_papers

\section{Recommended Citation}

Yu Wang and Jorge J. Santiago-Aviles, "Large negative magnetoresistance and strong localization in highly disordered electrospun pregraphitic carbon nanofiber", . September 2006.

Postprint version. Published in Applied Physics Letters, Volume 89, Issue 12, Article 123119, September 2006, 3 pages.

Publisher URL: http://dx.doi.org/10.1063/1.2338573

This paper is posted at ScholarlyCommons. https://repository.upenn.edu/ese_papers/212

For more information, please contact repository@pobox.upenn.edu. 


\title{
Large negative magnetoresistance and strong localization in highly disordered electrospun pregraphitic carbon nanofiber
}

\author{
Abstract \\ A highly disordered pregraphitic carbon nanofiber with the product of its quasi-Fermi wave vector and \\ mean free path close to 1 was fabricated using electrospinning technique. Strong localization made the \\ conductivity vary with temperature as $\sigma$ [proportional to] $T^{-1 / 2}$ from 300 to $5 \mathrm{~K}$, suggesting variable range \\ hopping as the conductivity mechanism, and resulted in a large negative magnetoresistance from $300 \mathrm{~K}$ \\ down to $1.9 \mathrm{~K}$ that can still be quantitatively described using weak localization and electron interaction \\ models.

\section{Comments} \\ Postprint version. Published in Applied Physics Letters, Volume 89, Issue 12, Article 123119, September \\ 2006, 3 pages. \\ Publisher URL: http://dx.doi.org/10.1063/1.2338573
}




\title{
Large negative magnetoresistance and strong localization in highly disordered electrospun pregraphitic carbon nanofiber
}

\author{
Yu Wang ${ }^{a)}$ and Jorge J. Santiago-Avilés \\ Department of Electrical and Systems Engineering, University of Pennsylvania, 200 South 33rd Street, \\ Philadelphia, Pennsylvania 19104
}

(Received 24 March 2006; accepted 18 July 2006; published online 21 September 2006)

\begin{abstract}
A highly disordered pregraphitic carbon nanofiber with the product of its quasi-Fermi wave vector and mean free path close to 1 was fabricated using electrospinning technique. Strong localization made the conductivity vary with temperature as $\ln \sigma \propto T^{-1 / 2}$ from 300 to $5 \mathrm{~K}$, suggesting variable range hopping as the conductivity mechanism, and resulted in a large negative magnetoresistance from $300 \mathrm{~K}$ down to $1.9 \mathrm{~K}$ that can still be quantitatively described using weak localization and electron interaction models. (C) 2006 American Institute of Physics. [DOI: 10.1063/1.2338573]
\end{abstract}

Disorder in electronic system is known to produce nonperiodic Anderson potentials. ${ }^{1}$ For weak disorder and small Anderson potential, their effect is to introduce a finite mean free path $l$. For strong disorder and Anderson potential, however, the electron is localized. Localization not only severely shortens the mean free path, but also gives rise to mobility edge $E_{c}$ in the conduction band. ${ }^{1,2}$ The product $k_{F} l$, where $k_{F}$ is the quasi-Fermi wave vector, reflects the degree of the disorder and localization. For $k_{F} l \gg 1$ disorder and localization are weak, quasi-Fermi level $E_{F}$ is close to $E_{c}$, and localized electrons can be thermally activated to the conducting band higher than $E_{c}$, where they can move by diffusion. Scaling theory predicts the logarithmic relation

$$
\sigma(T)=\sigma_{0}+\frac{p}{2} \frac{e^{2}}{\pi^{2} h} \ln \frac{T}{T_{0}},
$$

where $\sigma_{0}, p$, and $T_{0}$ are constants depending on the scattering mechanism, ${ }^{3}$ and negative magnetoresistance (MR) or equivalently, positive magnetoconductance (MC) in a twodimensional (2D) weakly localized system

$$
\begin{aligned}
G(B, T)-G(0, T)= & \frac{e^{2}}{\pi h}\left[\frac{3}{2} \Psi\left(\frac{1}{2}+\frac{B_{2}}{B}\right)-\Psi\left(\frac{1}{2}+\frac{B_{1}}{B}\right)\right. \\
& \left.-\frac{1}{2} \Psi\left(\frac{1}{2}+\frac{B_{3}}{B}\right)-\ln \frac{B_{2}^{3 / 2}}{B_{1} B_{3}^{1 / 2}}\right]
\end{aligned}
$$

where $G(B, T)$ is the conductance, $B$ is the magnetic field, $\Psi$ is the digamma function, and $B_{1}, B_{2}$, and $B_{3}$ are parameters characteristic of the fibers. ${ }^{3}$ Equation (2) originates from the field induced dephasing effect of quantum diffusive electron wave in the weak localization (WL) regime, and is therefore considered valid only for WL.

For $k_{F} l$ close to 1 , the disorder and electron localization are strong and localized electrons cannot be always thermally excited over their nearest neighboring energy barriers to the conducting band, and may have to hop to another localized state in a variable range. Mott, ${ }^{2}$ implicitly supposing no Coulomb interaction between localized electrons, derived the variable-range hopping $(\mathrm{VRH})$ conductivity as

\footnotetext{
${ }^{a)}$ Electronic mail: wangyu@ seas.upenn.edu
}

$$
\sigma(T)=\sigma_{M} \exp \left[-\left(\frac{T_{M}}{T}\right)^{1 /(d+1)}\right]
$$

with

$$
T_{M}=\frac{24}{\pi k_{B} \xi^{3} N\left(E_{F}\right)},
$$

where $\sigma_{M}$ is a constant, $d$ is the dimensionality, $k_{B}$ is the Boltzmann constant, $\xi$ is the localization distance, and $N\left(E_{F}\right)$ is the density of state (DOS) at $E_{F}$. Efros and Shklovskii ${ }^{4}$ further established that, if the Coulomb interaction between electrons is strong enough to create a "soft" gap in the DOS near $E_{F}$, the VRH conductivity is $d$-independent

$$
\sigma(T)=\sigma_{\mathrm{ES}} \exp \left[-\left(\frac{T_{\mathrm{ES}}}{T}\right)^{1 / 2}\right],
$$

with

$$
T_{\mathrm{ES}}=\frac{e^{2}}{\pi \varepsilon_{0} \varepsilon_{r} k_{B} \xi},
$$

where $\sigma_{\mathrm{ES}}$ is a constant, $\varepsilon_{0}$ is the permittivity of vacuum, and $\varepsilon_{r}$ is the dielectric constant in the disordered environment. Electron interaction effects also frequently cause negative MC through Zeeman spin splitting and orbital effect. Due to electron intrastate correlation, MC depends linearly on $B$ at low $B$ and saturates at higher $B .^{5}$

Strictly speaking, the Mott and Efros-Shklovskii (E-S) models are not based on the scaling theory. There is no well established scaling theory for electron transport in the strong localization case of $k_{F} l \sim 1$. Minkov et al. ${ }^{6}$ recently found that Eqs. (1) and (2), although derived on the condition of $k_{F} l \gg 1$, can be applied in a strongly localized 2D electron gas with $k_{F} l=0.5-2$. Lee et al. ${ }^{7}$ also showed the validity of Eq. (2) beyond the WL regime.

Versatile, conductive carbons are good disordered electron systems to explore, in that they are inexpensive and easily available and their disorder can be tuned easily by their fabrication parameters. Since its discovery in pregraphitic carbon in $1956,{ }^{8}$ negative MR has been found not only in the partially ordered bulk carbon, ${ }^{9}$ but also in carbon thin film, ${ }^{10}$ carbon fibers, and carbon microcoils, no matter whether they were spun from polyacrylonitrile (PAN), ${ }^{11}$ benzene, ${ }^{12}$ pitch, ${ }^{13}$ or by chemical vapor deposition. ${ }^{14}$ Recently, there are many reports on the electro- and magneto- 


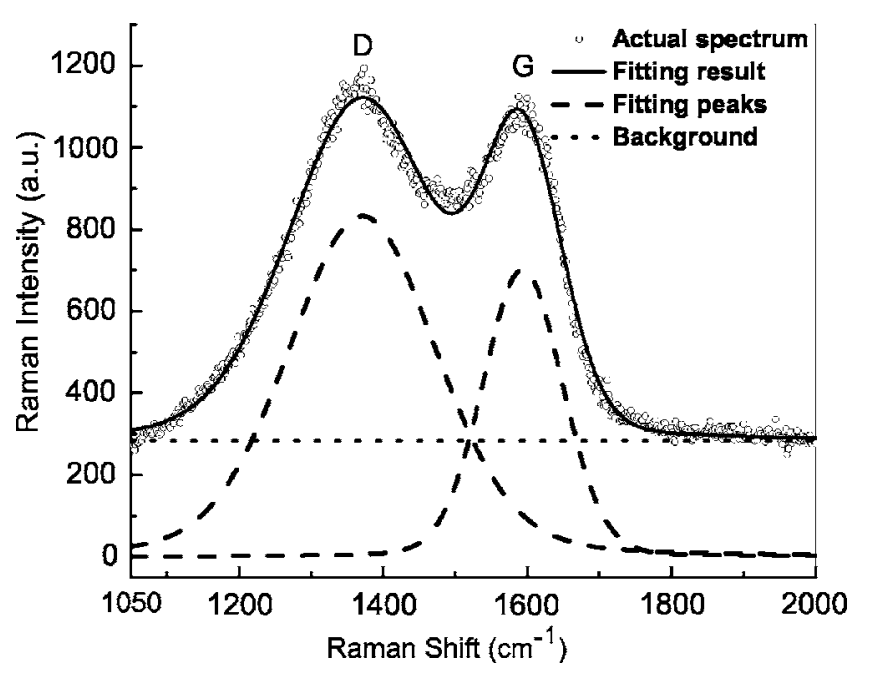

FIG. 1. Typical Raman spectrum of fibers treated at $1173 \mathrm{~K}$.

transport in carbon nanotubes. ${ }^{15,16}$ Irrespective of the dimensionality, negative MR was found directly related to the disorder in structure. However, there are few such reports in carbon nanofibers explicitly in the strong localization regime. Bayot et al. ${ }^{17}$ graphitized carbon microfibers and sheets at and above $2000{ }^{\circ} \mathrm{C}$ so that $k_{F} l \gg 1$. We found that large negative MR follows Eq. (2) in a highly disordered electrospun pregraphitic carbon nanofiber heat treated in vacuum at $1273 \mathrm{~K}^{18}$ The electron density of that fiber, $n$, was measured to be about $2 \times 10^{25} \mathrm{~m}^{-3},{ }^{19}$ and its $k_{F}$ $\approx\left(3 \pi^{2} n\right)^{1 / 3} \approx 8 \times 10^{8} \mathrm{~m}^{-1}$. Taking its graphitic crystallite size $L_{a}=2.5 \mathrm{~nm}$ (Ref. 20) as a convenient and good approximation to $l$, its $k_{F} l$ is no larger than 2, implying a regime close to strong localization. By decreasing the vacuum heat treatment temperature (HTT) to $1173 \mathrm{~K}$, this letter further enhanced the disorder to a degree of $k_{F} l \sim 1$ and probed the electro- and magnetotransport in the strong localized carbon nanofiber.

The precursor is a solution of commercial PAN in N, $\mathrm{N}$-dimethyl formamide (DMF) with a ratio of $6 \mathrm{~g}$ PAN to $100 \mathrm{ml}$ DMF. The substrates were thermally oxidized silicon wafers with their $150 \mathrm{~nm}$ surface oxide lithography patterned with a $1 \times 1 \mathrm{~mm}^{2}$ gold contact array. A homemade electrospinning setup, described elsewhere, ${ }^{20,21}$ was used to spin single precursor fibers between four isolated gold contacts in a row. The as-spun fibers were heat treated at $1173 \mathrm{~K}$ for $30 \mathrm{~min}$ in a vacuum of $10^{-6}$ Torr. The treated fibers were characterized at $300 \mathrm{~K}$ using a Renishaw Raman microspectrometer with its exciting laser wavelength $=514 \mathrm{~nm}$.

Four-probe conductance $(G)$ measurements were conducted using model 6000 physical properties measurement system by Quantum Design Inc., equipped with a Keithley 237 high voltage source measurement unit. The measuring power was maintained at $10^{-8} \mathrm{~W}$ to suppress self-heating. The temperature was first swept from 300 to $1.9 \mathrm{~K}$. Then, transverse magnetic field was applied and swept between -9 and $9 \mathrm{~T}$ (negative sign stands for the reverse direction of the magnetic field), back and forth, at 300.0, 10.0, 5.0, 3.0, and $1.9 \mathrm{~K}$, respectively. The length $L$ and cross-sectional area $S$ of the fibers were measured using scanning electron and scanning probe microscopes. ${ }^{20,21}$

In the Raman scattering spectrum (Fig. 1), two broad overlapping peaks are centered around 1360 and $1590 \mathrm{~cm}^{-1}$ and attributed to the disordered $(D)$ and graphite $(G)$ carDownloaded 07 Dec 2006 to 130.91.116.168. Redistribution subje
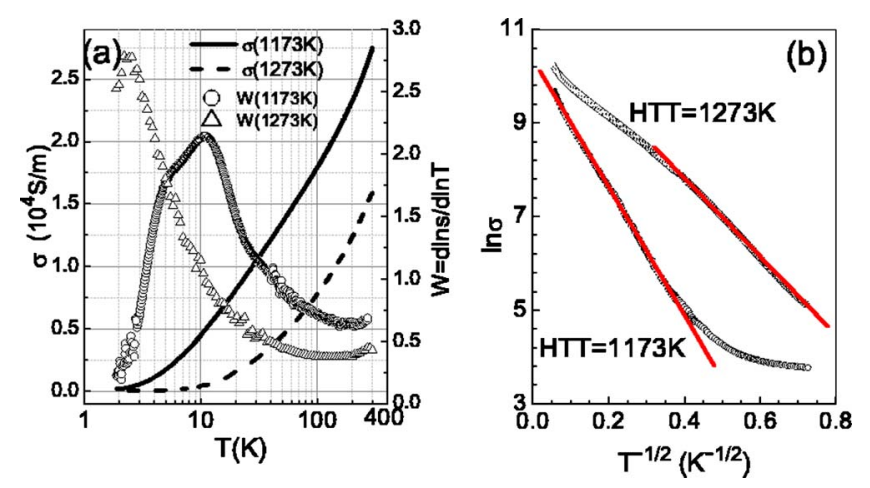

FIG. 2. (Color online) Temperature dependence of conductivity of the carbon nanofiber.

bons, respectively. They were fitted to a Gaussian-Lorentzian shape, with their integrated intensity ratio $R=2.1$. The inplane graphitic crystallite size $L_{a}$ was estimated to be about $2 \mathrm{~nm}$, using the relation put forward by Knight and White ${ }^{22}$ of $L_{a}(\mathrm{~nm})=4.4 / R$. The fibers heat treated at $1273 \mathrm{~K}$ have a similar Raman spectrum with $R=1.8$ and $L_{a}=2.5{ }^{20}$ The lower $L_{a}$ value and the higher reduced resistivity $\rho(1.9 \mathrm{~K}) / \rho(300 \mathrm{~K})[$ Fig. $2(\mathrm{a})]$ for HTT $=1173 \mathrm{~K}$ confirm the disorder enhancement by the decrease of HTT.

Since MC is positive for HTT $=1173 \mathrm{~K}$, the carrier mobility cannot be measured from the negative parabolic field dependence of MC as in the case of HTT $=1273 \mathrm{~K},{ }^{19}$ and the $n$ value is unknown for the time being. However, $n$ and $k_{F} l$ values can still be estimated. At room temperature, $\sigma$ value for HTT $=1173 \mathrm{~K}$ is only half of that for HTT $=1273 \mathrm{~K}$ [Fig. 2(a)]. If the ratio is attributed exclusively to the difference in $n, n$ value for HTT $=1173 \mathrm{~K}$ will be half of that for HTT $=1273 \mathrm{~K},{ }^{19}$ i.e., $10^{25} \mathrm{~m}^{-3}, k_{F} \approx\left(3 \pi^{2} n\right)^{1 / 3} \approx 6 \times 10^{8} \mathrm{~m}^{-1}$, and its $k_{F} l \approx k_{F} L_{a}$ is 1.2 .

While the fiber treated at $1273 \mathrm{~K}$ follows Eq. (1) between 10 and $200 \mathrm{~K}$, manifesting a WL regime [the deviation from Eq. (1) between 200 and $300 \mathrm{~K}$ may be attributed to some nonlocalized electrons in the latter fiber], that is not the case for the fiber treated at $1173 \mathrm{~K}$, where the linear relation between $\ln \sigma(T)$ and $T^{1 / 2}$ [Fig. 2(b)] confirms the VRH regime between 300 and $5 \mathrm{~K}$. Although such a relation cannot identify whether the VRH regime is of the Mott type (with $d=1$ ) or the E-S type, the turbostratic phase structure of our pregraphitic carbon fibers demands $d=2,{ }^{17}$ and $\sigma(T)$ follows Eq. (4a) with $T_{\mathrm{ES}}=187 \mathrm{~K}$. Using $\xi \approx L_{a} \approx 2 \mathrm{~nm}$ and Eq. (4b), we can estimate $\varepsilon_{r} \approx 200$. This value seems reasonable in that it lies between the metallic and insulating limits of $\varepsilon_{r} \approx \infty$ and $\varepsilon_{r} \approx 10$, respectively.

However, the strong temperature dependence of $\sigma(T)$ below $5 \mathrm{~K}$ does not fit Eq. (1), (3), or (4). To obtain a quantitative insight into that cause, the reduced activation energy $W \equiv d \ln \sigma(T) / d \ln T$ is shown in Fig. 2(a). While $W$ increases with the decrease of temperature showing an insulating regime above $11 \mathrm{~K}$, it decreases with the temperature showing a metallic regime below $11 \mathrm{~K}$. The transition around $11 \mathrm{~K}$, whose reason remains to be clarified, may account for the deviation of $\sigma(T)$ from Eqs. (1), (3), and (4).

The carbon nanofiber heat treated at $1173 \mathrm{~K}$ manifests positive $\mathrm{MC}$ in the whole investigated temperature and magnetic field ranges except for the small negative MC under low magnetic field at $1.9 \mathrm{~K}$ (Fig. 3). In contrast, the heat treatment at $1273 \mathrm{~K}$ resulted in positive MC at temperatures to AIP license or copyright, see http://apl.aip.org/apl/copyright.jsp 


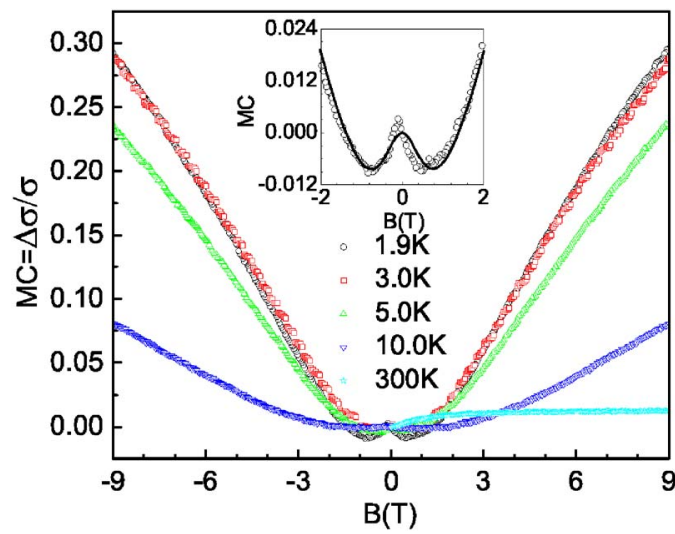

FIG. 3. (Color online) Field dependence of magnetoconductance.

lower than $10 \mathrm{~K}$. Around $10 \mathrm{~K}, \mathrm{MC}$ is negative under low magnetic field and positive under high magnetic field. ${ }^{18}$ Irrespective of HTT, positive MC corresponds to temperature dependence of conductivity as Eq. (4), and negative MC corresponds to a deviation from Eq. (4). Although a small negative $\mathrm{MC}$ is often attributed to spin-orbit interaction, ${ }^{23}$ such an effect is trivial in carbon. Instead, the effect of electronelectron $(e-e)$ interaction seems more appropriate because the E-S-type VRH indicates strong $e-e$ interaction. Using the formula by Frydman and Ovadyahu ${ }^{24}$ and considering $B_{2}$ $=B_{3}$ (due to weak magnetic scattering and spin-orbit interaction in carbon), Eq. (2) is modified as

$$
\begin{aligned}
\mathrm{MC}= & \eta(T)\left[\Psi\left(\frac{1}{2}+\frac{B_{2}}{B}\right)-\Psi\left(\frac{1}{2}+\frac{B_{1}}{B}\right)-\ln \frac{B_{2}}{B_{1}}\right] \\
& -A_{e} \frac{B^{2}}{B^{2}+B_{e}^{2}},
\end{aligned}
$$

where $\eta(T)$ and $A_{e}$ are constants, $B_{e}$ is a characteristic field for spin alignment, and the second term on the right hand side is the contribution of $e-e$ interaction. Nonlinear curve fitting indicates that the MC fits Eq. (5) very well between 1.9 and $300 \mathrm{~K}$. Of the fitting results (Table I), the small $A_{e}$ values indicate that the $e$-e contribution to the MC (negative) is much smaller in magnitude than that of WL (positive). Although it is unexpected that MC still mainly follows Eq. (2) even in strong localization, a careful review on its derivation gave us a plausible explanation. In that derivation, ${ }^{3}$ $\beta(G) \equiv d \ln G / d \ln L$ was expanded as

$$
\beta=(d-2)+b_{1} G^{-1}+b_{2} G^{-2},
$$

where the zero-order term $d-2=0$ in a 2D case, the firstorder term leads to Eq. (2), and $b_{2}=0$ in the "orthogonal

TABLE I. Fitting parameters.

\begin{tabular}{ccccc}
\hline \hline \multicolumn{1}{c}{$T$} & $1.9 \mathrm{~K}$ & $3.0 \mathrm{~K}$ & $5.0 \mathrm{~K}$ & $10.0 \mathrm{~K}$ \\
\hline$A_{e} \pm \Delta A_{e}(T)$ & $0.0290 \pm 0.001$ & $0.024 \pm 0.003$ & $0.011 \pm 0.001$ & $0.028 \pm 0.011$ \\
$B_{1} \pm \Delta B_{1}(T)$ & \multicolumn{4}{c}{$4.133 \pm 0.653$} \\
$B_{2} \pm \Delta B_{2}(T)$ & $1.702 \pm 0.1650$ & $1.603 \pm 0.170$ & $2.347 \pm 0.256$ & $4.093 \pm 3.278$ \\
$B_{e} \pm \Delta B_{e}(T)$ & $0.687 \pm 0.035$ & $1.017 \pm 0.076$ & $0.560 \pm 0.054$ & $3.063 \pm 0.434$ \\
$\eta \pm \Delta \eta$ & $1.141 \pm 0.295$ & $1.002 \pm 0.251$ & $1.620 \pm 0.724$ & $54.240 \pm 4990.65$ \\
\hline \hline
\end{tabular}

case." Equation (6) is equivalent to the expansion of $2 \mathrm{D}$ conductivity by Gor'kov et $\mathrm{al}^{25}$ as

$$
\begin{aligned}
\sigma_{2 \mathrm{D}}(L)= & \sigma_{0}\left[1-c_{1}\left(k_{F} l\right)^{-1} \ln (L / l)+c_{2}\left(k_{F} l\right)^{-2} \ln ^{2}(L / l)\right. \\
& +\cdots],
\end{aligned}
$$

where the coefficient $c_{2}$ vanishes in 2D. Both equations indicate that the correction is a third-order term and may still be very small even in a strong localization regime.

In summary, a highly disordered pregraphitic carbon nanofiber with partial nanoscale graphitic crystallites was derived from PAN by electrospinning and heat treatment at $1173 \mathrm{~K}$. The fiber is so disordered that its $k_{F} l$ value is close to 1 and its conduction is in the strong localization regime. Its conductivity decreases with the measuring temperature from $300 \mathrm{~K}$ down to $1.9 \mathrm{~K}$, following the Efros-Shklovskii type of VRH conduction between 300 and $5 \mathrm{~K}$. The fiber manifested large negative MR between 1.9 and $300 \mathrm{~K}$, except for the small positive MR at $1.9 \mathrm{~K}$ and $B<1 \mathrm{~T}$. The MR still mainly follows the model of $2 \mathrm{D}$ WL plus small correction from $e$-e interaction.

The authors thank Jey Kikawwa from Department of Physics at the University of Pennsylvania for his help in conductivity measurements.

${ }^{1}$ P. W. Anderson, Phys. Rev. 109, 1492 (1958)

${ }^{2}$ N. F. Mott and E. A. Davis, Electronic Processs in Non-Crystalline Materials (Clarendon, Oxford, 1971), 2nd ed. (Clarendon, Oxford, 1979).

${ }^{3}$ S. Hikami, A. I. Larkin, and Y. Nagaoka, Prog. Theor. Phys. 63, 707 (1980).

${ }^{4}$ A. L. Efros and B. I. Shklovskii, J. Phys. C 8, L49 (1975).

${ }^{5}$ A. Kurbe and H. Kamimura, J. Phys. Soc. Jpn. 50, 2561 (1981).

${ }^{6}$ G. M. Minkov, O. E. Rut, A. V. Germanenko, and A. A. Sgerstobitov, Phys. Rev. B 65, 235322 (2002).

${ }^{7}$ Y. J. Lee, Y. S. Kim, and H. K. Shin, J. Phys.: Condens. Matter 14, 483 (2002).

${ }^{8}$ S. Mrozowski and A. Chaberski, Phys. Rev. 104, 74 (1956).

${ }^{9}$ Y. Hishiyama, Carbon 8, 259 (1976).

${ }^{10}$ A. Faißt and H. V. Löhneysen, Carbon 40, 321 (2002).

${ }^{11}$ D. Robson, F. Y. I. Assabghy, and D. J. E. Ingram, J. Phys. D 5, 169 (1972).

${ }^{12}$ M. Endo, Y. Hishiyama, and T. Koyama, J. Phys. D 15, 353 (1982).

${ }^{13}$ A. A. Bright and L. S. Singer, Carbon 17, 59 (1979).

${ }^{14}$ M. Fuji, M. Matsui, S. Motojima, and Y. Hishikawa, Thin Solid Films 409, 78 (2002).

${ }^{15}$ R. Tarkiainen, M. Ahlskog, A. Zyuzin, P. Hakonen, and M. Paalanen, Phys. Rev. B 69, 033402 (2004).

${ }^{16}$ J. Vavro, J. M. Kikkawa, and J. E. Fischer, Phys. Rev. B 71, 155410 (2005).

${ }^{17}$ V. Bayot, L. Piraux, J.-P. Michenaud, and J.-P. Issi, Phys. Rev. B 41, 11770 (1990).

${ }^{18}$ Yu Wang and J. J. Santiago-Avilés, J. Appl. Phys. 94, 1721 (2003).

${ }^{19}$ Jorge J. Santiago-Avilés and Yu Wang, Carbon 41, 2665 (2003).

${ }^{20}$ Y. Wang, S. Serrano, and J. J. Santiago-Aviles, Synth. Met. 138, 423 (2003).

${ }^{21}$ Y. Wang, J. J. Santiago-Aviles, R. Furlan, and I. Ramos, IEEE Trans. Nanotechnol. 2, 39 (2003).

${ }^{22}$ D. S. Knight and W. B. White, J. Mater. Res. 4, 385 (1989).

${ }^{23}$ B. I. Shklovskii and A. L. Efros, Electronic Properties of Doped Semiconductor, Solid State Science Vol. 45 (Springer, Berlin, 1984).

${ }^{24}$ A. Frydman and Z. Ovadyahu, Solid State Commun. 94, 745 (1995).

${ }^{25}$ L. P. Gor'kov, A. I. Larkin, and D. E. Khmel'nitskii, JETP Lett. 30, 248 (1979). 